ITP-UH-03/10

HWM-10-1

EMPG-10-01

\title{
UV/IR duality in noncommutative quantum field theory ${ }^{1}$
}

\author{
André Fischer \\ Institut für Theoretische Physik \\ Leibniz Universität Hannover \\ Appelstraße 2, D-30167 Hannover, Germany \\ Email: afischer@itp.uni-hannover.de \\ Richard J. Szabo \\ Department of Mathematics \\ Heriot-Watt University \\ Colin Maclaurin Building, Riccarton, Edinburgh EH14 4AS, U.K. \\ and Maxwell Institute for Mathematical Sciences, Edinburgh, U.K. \\ Email: R.J.Szabo@ma.hw.ac.uk
}

\begin{abstract}
We review the construction of renormalizable noncommutative euclidean $\phi^{4}$-theories based on the UV/IR duality covariant modification of the standard field theory, and how the formalism can be extended to scalar field theories defined on noncommutative Minkowski space.
\end{abstract}

\footnotetext{
${ }^{1}$ Based on invited talks given by the second author at the Bayrischzell Workshop on "Noncommutativity and Physics: Quantum Geometries and Gravity", Bayrischzell, Germany, May 15-18 2009; at the 2nd School on "Quantum Gravity and Quantum Geometry" session of the 9th Hellenic School on Elementary Particle Physics and Gravity, Corfu, Greece, September 13-20 2009; and at the Dublin Institute for Advanced Studies Workshop on "Noncommutativity and Matrix Models", Dublin, Ireland, November 23-27 2009. To be published in General Relativity and Gravitation.
} 


\section{Renormalization of noncommutative euclidean scalar field theory}

In this article we will review recent progress in understanding how to renormalize field theories on noncommutative euclidean space, and some new advances into how these models may be analytically continued to Minkowski signature. Here we will be exclusively interested in scalar field theories on Moyal spacetimes of even dimension. If $\phi$ is a real scalar field on $\mathbb{R}^{2 d}$ with Fourier transform $\widetilde{\phi}$, then the interactions in noncommutative field theory on this space can be encoded by modifying the pointwise products $\phi \cdot \phi$ to star-products $\phi \star \phi$, which in momentum space amounts to altering the Fourier convolution products as

$$
\widetilde{\phi}(k) \widetilde{\phi}(q) \longrightarrow \widetilde{\phi}(k) \widetilde{\phi}(q) \mathrm{e}^{\mathrm{i} k \times q}, \quad k \times q=\frac{1}{2} k_{\mu} \theta^{\mu \nu} q_{\nu}
$$

where $\theta^{\mu \nu}$ is a constant antisymmetric matrix which we assume is of maximal rank for simplicity. Foundational aspects of the theory are covered in [1].

In $\lambda \phi_{2 d}^{\star n}$-theory, the interaction vertex in momentum space is thus modified to

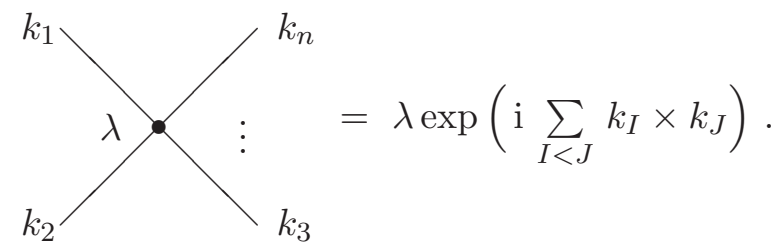

The perturbative quantum field theory suffers from the infamous UV/IR mixing problem [2]. While the convergence properties of planar graphs are the same as in the corresponding commutative quantum field theory at $\theta=0$, the phase factors in (1.2) drastically alter the properties of non-planar graphs. Although this phase factor improves the ultraviolet behaviour of amplitudes, divergences reappear as poles at vanishing external momenta. This can be summarized by saying that an ultraviolet cutoff $\Lambda$ necessarily induces an effective infrared cutoff $\Lambda_{0}=\frac{1}{\theta \Lambda}$. This would seem to ruin standard renormalization schemes, such as the wilsonian prescription which requires a clear separation of energy scales. More precisely, although at one-loop order the on-shell amplitudes for massive particles are all finite, when these graphs are inserted as sub-graphs at higher-loop orders virtual particles of vanishing momentum produce uncontrollable divergences in the amplitudes. Thus the field theory cannot be renormalized. UV/IR mixing also occurs on more complicated noncommutative spaces such as $\kappa$-deformed spaces [3], and thus appears to be a generic feature of noncommutative field theories.

The cure to this problem $[4,5]$ is to consider instead a covariant version of the field theory which renders its ultraviolet and infrared regimes indistinguishable. In momentum space, this can be regarded as a modification of the Fourier momenta as

$$
k_{\mu} \longmapsto K_{\mu}=k_{\mu}+B_{\mu \nu} x^{\nu},
$$

where $B_{\mu \nu}$ is another antisymmetric constant matrix of maximal rank which is generically independent of $\theta^{\mu \nu}$. We may think of this matrix as a "magnetic" background, so that the momenta (1.3) can be regarded as "Landau" momenta. If $\left(k_{\mu}, x^{\nu}\right)$ are canonically conjugate variables, then these new momenta generate a "noncommutative momentum space",

$$
\left[K_{\mu}, K_{\nu}\right]=2 \text { i } B_{\mu \nu}
$$

the familiar feature of physical momenta for charged particles propagating in a constant magnetic field. Thus the position and momentum spaces are formally identical, and there is no longer any distinction between what is meant by ultraviolet or infrared. 
The Grosse-Wulkenhaar model is then the real euclidean scalar $\lambda \phi_{2 d^{-}}^{\star 4}$ theory in a background harmonic oscillator potential. Analogously to (1.3), this amounts to replacing the Laplace operator giving the kinetic term in the scalar field action according to

$$
\partial_{\mu}^{2} \longmapsto \partial_{\mu}^{2}+\frac{\omega^{2}}{2} \widetilde{x}_{\mu}^{2}, \quad \widetilde{x}_{\mu}=2 \theta_{\mu \nu}^{-1} x^{\nu}
$$

The quantum field theory is then symmetric under Fourier transformation of fields, which amounts to exchanging momenta with positions as $k_{\mu} \leftrightarrow \widetilde{x}_{\mu}$.

The renormalization properties of this modified noncommutative field theory may then be summarized as follows [5]-[9]. The covariant model is renormalizable to all orders in $\lambda$. A crucial ingredient of the original renormalizability proof [5] is the fact that the quantum field theory can regulated and described by a matrix model, with natural cutoff the matrix size $N$. At $\omega=1$ the field theory is completely invariant under the UV/IR duality transformation, without any rescalings of the parameters, whence this point in parameter space is called the self-dual point. At this point, the beta-functions in both couplings $\lambda$ and $\omega$ vanish to all orders of perturbation theory, and thus the renormalized coupling flows to a finite bare coupling. This is analogous to what happens in a conformally invariant quantum field theory. It implies, in particular, that the duality covariant noncommutative field theory contains no Landau ghost (or renormalons), contrary to the usual commutative $\phi_{4}^{4}$-theory, and unlike non-abelian gauge theories this elimination is achieved without asymptotic freedom (but instead with "asymptotic safety"). For these reasons, a non-perturbative completion of the quantum field theory is believed possible. See [8] for further details.

\section{$2 \mathrm{UV} / \mathrm{IR}$ duality on noncommutative euclidean space}

In this article we will demonstrate how to construct an analogous duality covariant scalar field theory on noncommutative Minkowski space. For later comparison, and because some of the duality proofs transcribe immediately to lorentzian signature, we will first review the proof of euclidean duality in some detail and the ensuing matrix model representation, following $[4,6]$. The Grosse-Wulkenhaar model has also been formulated on solvable symmetric spaces in [10], where the UV/IR duality is interpreted in terms of metaplectic representations of the Heisenberg group.

\subsection{Classical duality}

The original duality covariant model considers charged scalar fields $\phi(x)$ on euclidean space $\mathbb{R}^{2 d}$ with action

$$
S[\phi]=\int \mathrm{d}^{2 d} x\left(\phi^{\dagger}\left(D_{\mu}^{2}+\mu^{2}\right) \phi+g^{2} \phi^{\dagger} \star \phi \star \phi^{\dagger} \star \phi\right),
$$

where

$$
D_{\mu}=\frac{1}{\sqrt{2}}\left(-\mathrm{i} \partial_{\mu}+B_{\mu \nu} x^{\nu}\right)
$$

is the gauge-covariant derivative in the magnetic background. All of our considerations also apply to the other inequivalent quartic interaction $\phi^{\dagger} \star \phi^{\dagger} \star \phi \star \phi$, but for simplicity we focus only on the one given in (2.1). This action is invariant under the duality transformation of order two given by

$$
\begin{aligned}
\phi(x) & \longrightarrow \widehat{\phi}(x)=\sqrt{|\operatorname{det}(B)|} \tilde{\phi}(B \cdot x), \\
\theta & \longrightarrow \widehat{\theta}=-4 B^{-1} \theta^{-1} B^{-1} \\
g & \longrightarrow \widehat{g}=2^{d}|\operatorname{det}(B \theta)|^{-1 / 2} g
\end{aligned}
$$


The proof is an elementary, but somewhat lengthy, calculation [4]. The statement for the quadratic terms is a straightforward application of the Parseval identity, while for the quartic terms it follows from the integral kernel representation of the star-product (valid on the space of Schwartz fields $\phi)$. With the normalizations above the self-dual point, where the action is completely invariant on parameter space, is given by $\theta=2 B^{-1}$.

\subsection{Quantum duality}

While the UV/IR duality is relatively straightforward at the classical level, it is somewhat more involved at the quantum level. The quantum field theory is defined by the generating functional of connected Green's functions

$$
\mathcal{G}(J)=-\log \frac{Z[J]}{Z[0]}
$$

where $Z[J]$ is the partition function for the field theory coupled to external sources $J$ and $J^{\dagger}$ given by the functional integral

$$
Z[J]=\int \mathcal{D} \phi \mathcal{D} \phi^{\dagger} \exp \left(-S[\phi]-\int \mathrm{d}^{2 d} x\left(\phi^{\dagger} J+\phi J^{\dagger}\right)\right) .
$$

Correlation functions are obtained by taking suitable functional derivatives with respect to $J$ and $J^{\dagger}$. By Parseval's identity, the extra source couplings in (2.5) are invariant under (2.3), and so is the functional integration measure. Thus the generating functional is formally invariant under the duality transformation of Schwartz functions $\phi \longmapsto \widehat{\phi}$ on $\mathbb{R}^{2 d}$,

$$
\mathcal{G}(J ; B, g, \theta)=\mathcal{G}(\widehat{J} ; B, \widehat{g}, \widehat{\theta}) .
$$

However, to substantiate this claim we need to make sense of the functional integration in (2.5), which nessitates regulated perturbation theory. This requires a duality invariant regularization $\mathcal{G} \longrightarrow \mathcal{G}_{\Lambda}$ which we now describe.

For simplicity, let us consider the case $d=1$. The crux of the proof is to expand the scalar fields not in the standard plane wave eigenstates of the momentum operator $-\mathrm{i} \partial_{\mu}$ (which requires distinguishing between ultraviolet and infrared), but instead in the "matrix basis" $f_{n, m} \in L^{2}\left(\mathbb{R}^{2}\right)$, $n, m=0,1, \ldots$ of Landau wavefunctions

$$
\phi(x)=\sum_{n, m=0}^{\infty} f_{n, m}(x) \phi_{n, m},
$$

which are eigenstates of the Landau hamiltonian with

$$
D_{\mu}^{2} f_{n, m}=2 B\left(n+\frac{1}{2}\right) f_{n, m}=: E_{n} f_{n, m} .
$$

Note that each Landau level is infinitely degenerate. The second quantum number features because the Landau wavefunctions are simultaneously eigenstates of the Landau hamiltonian with the reflected magnetic field,

$$
\left.D_{\mu}^{2}\right|_{B \rightarrow-B} f_{n, m}=E_{m} f_{n, m},
$$

such that the sum $D_{\mu}^{2}+\left.D_{\mu}^{2}\right|_{B \rightarrow-B}=-\partial_{\mu}^{2}+(B \cdot x)_{\mu}^{2}$ gives the background harmonic oscillator potential which figures in the Grosse-Wulkenhaar model.

For a suitable cut-off function $F:[0, \infty) \rightarrow[0, \infty)$, with $F(0)=1$ and $F(s)=0$ for all $s>s_{0}$ and some finite $s_{0}$, we now replace the free propagator in the Landau basis,

$$
C(n, m)=\left(E_{n}+\mu^{2}\right)^{-1}
$$


with the regulated propagator

$$
C_{\Lambda}(n, m)=\left(E_{n}+\mu^{2}\right)^{-1} F\left(\Lambda^{-2}\left(E_{n}+E_{m}\right)\right)
$$

where $\Lambda \in \mathbb{R}$ provides an ultraviolet cutoff in $(n, m)$-space and $C_{\Lambda} \rightarrow C$ as $\Lambda \rightarrow \infty$. The argument of the cut-off function is the eigenvalue of the differential operator $D_{\mu}^{2}+\left.D_{\mu}^{2}\right|_{B \rightarrow-B}$. In particular, the cut-off on the operator $-\partial_{\mu}^{2}$ truncates all high-momentum modes while the cut-off on the operator $(B \cdot x)_{\mu}^{2}$ truncates the long-distance modes.

Since $F(s)=0$ for $s>s_{0}$ and some finite $s_{0} \in(0, \infty)$, for $\Lambda$ finite the propagators $(2.11)$ are non-zero only for $E_{n}+E_{m}$ smaller than a uniform upper bound, which from the forms of the Landau eigenvalues can happen only for finitely many values of $n, m$. With this regularization, every Feynman diagram is of the schematic form

$$
\sum_{n_{1}, m_{1}, \ldots, n_{K}, m_{K} \geq 0} \prod_{k=1}^{K} C_{\Lambda}\left(n_{k}, m_{k}\right) \times \text { (vertices) }
$$

where the vertex factors will in general be complicated combinatorial quantities, but their explicit form is immaterial for our argument. Since the propagators in (2.12) are non-zero for only finitelymany $n_{k}, m_{k}$, every Feynman diagram is given by a finite sum, i.e. all Feynman amplitudes converge. This completes the proof of quantum duality.

\subsection{Matrix model representation}

A beautiful feature of the covariant quantum field theory is that it can be mapped exactly onto a matrix model, a manipulation which has no counterpart in ordinary field theory. For simplicity, we consider the two-dimensional model at the self-dual point, $d=1$ and $\theta=2 B^{-1}>0$ (see [6] for the general case). The crucial feature is the projector property of the Landau wavefunctions with respect to the star-product,

$$
f_{n, m} \star f_{n^{\prime}, m^{\prime}}=\sqrt{\frac{B}{4 \pi}} \delta_{m, n^{\prime}} f_{n, m^{\prime}},
$$

together with $f_{n, m}{ }^{*}=f_{m, n}$ and the normalization

$$
\int \mathrm{d}^{2} x f_{n, m}(x)=\sqrt{\frac{4 \pi}{B}} \delta_{n, m} .
$$

The action functional (2.1) can thereby be expressed in the form

$$
S[\phi]=\operatorname{Tr}\left(\phi^{\dagger} \mathcal{B} \phi+\mu^{2} \phi^{\dagger} \phi+\tilde{g}^{2}\left(\phi^{\dagger} \phi\right)^{2}\right)
$$

where in this formula $\phi=\left(\phi_{n, m}\right)$ is an infinite matrix, $\tilde{g}^{2}=B g^{2} / 4 \pi$, and $\mathcal{B}_{n, m}=2 B\left(n+\frac{1}{2}\right) \delta_{n, m}$. The quantum field theory thus has a $U(\infty)$ symmetry $\phi \longrightarrow U^{\dagger} \phi U$, and is the $N \longrightarrow \infty$ limit of the $N \times N$ complex matrix model in an external field whose partition function is given by

$$
Z_{N}=\int \prod_{n, m=1}^{N} \mathrm{~d} \phi_{n, m} \mathrm{~d} \phi_{n, m}^{\dagger} \mathrm{e}^{-S[\phi]} .
$$

This is an integral over a finite-dimensional space, and thus gives a constructive non-perturbative definition of the quantum field theory. Various exact integrability properties of the model in this representation, which is related to the Kontsevich-Penner matrix model (a hermitean matrix model in an external field with logarithmic potential), are described in [6]. 


\section{Analytic continuation to Minkowski signature}

The continuation of the duality covariant field theory to noncommutative Minkowski space is naively obtained by Wick rotation $x^{0} \longrightarrow \pm \mathrm{i} t$ plus an additional change $B_{0 i} \longrightarrow \pm \mathrm{i} E_{i}$, giving the dynamics in an electromagnetic background. While this is wrong for a number of reasons, we shall see that many of our results can be obtained in hindsight via a careful continuation of this sort. The reason that this naive approach is not expected to work is that the perturbative dynamics of (noncovariant) noncommutative field theory cannot be obtained simply by Wick rotation [11]-[13]. In contrast to the commutative case, time-ordering factors and the two-point function do not combine into Feynman propagators in non-planar graphs with a noncommuting time direction. Because of this complication, the Dyson and Feynman expansions are distinct, and the renormalization properties in the Dyson series are very different. By developing the complicated rules of timeordered perturbation theory on noncommutative spacetime, one can restore unitarity and causality of the quantum field theory. In fact, it has been suggested that UV/IR mixing may be far less severe or even absent in this case [14]. In the following we shall summarize results from the analysis of [15] which are carried out by defining the quantum field theory using a functional integral framework, rather than time-ordered perturbation theory.

\subsection{Results}

We will begin by stating the main results from [15], before going into the technical details of their derivation, which requires various notions from functional analysis and the theory of generalized functions. Again we restrict to the case of $1+1$ dimensions, corresponding to a pure electric background, the general case being a straightforward combination with the earlier euclidean analysis [15].

\section{$\underline{\text { Matrix basis }}$}

There is a dense domain of scalar fields $\phi \in \Phi \subset L^{2}\left(\mathbb{R}^{2}\right)$ and "electric Landau wavefunctions" $f_{n, m}^{ \pm} \in \Phi^{\prime}, n, m=0,1, \ldots$ such that

$$
\phi(x)=\sum_{n, m=0}^{\infty} f_{n, m}^{+}(x) \phi_{n, m}^{-}=\sum_{n, m=0}^{\infty} f_{n, m}^{-}(x) \phi_{n, m}^{+},
$$

and

$$
D_{\mu}^{2} f_{n, m}^{ \pm}= \pm \mathrm{i} E_{n} f_{n, m}^{ \pm},\left.\quad D_{\mu}^{2}\right|_{B \rightarrow-B} f_{n, m}^{ \pm}= \pm \mathrm{i} E_{m} f_{n, m}^{ \pm} .
$$

The \pm labels here correspond to the two choices of sign in the Wick rotation. In fact, although we shall not derive them in this way, the wavefunctions $f_{n, m}^{ \pm}$can be obtained merely by Wick rotating the standard Landau wavefunctions. Nevertheless, this result should look somewhat odd to the reader, since (3.2) seems to assert that $f_{n, m}^{ \pm}$are eigenfunctions of self-adjoint operators with imaginary eigenvalues. However, the crucial point is that these functions live in the topological dual $\Phi^{\prime}$ of the domain $\Phi$, which is much larger than the domain of these differential operators. Below we will see how this can be used to define notions of generalized eigenfunctions with generalized eigenvalues, which can be complex. The electric Landau wavefunctions obey the $L^{2}$-orthonormality and star-product projector relations

$$
f_{n, m}^{ \pm}{ }^{*}=f_{m, n}^{\mp}, \quad\left(f_{n, m}^{ \pm}, f_{n^{\prime}, m^{\prime}}^{\mp}\right)_{L^{2}}=\delta_{m, n^{\prime}} \delta_{n, m^{\prime}}, \quad f_{n, m}^{ \pm} \star f_{n^{\prime}, m^{\prime}}^{ \pm}=\sqrt{\frac{B}{4 \pi}} \delta_{m, n^{\prime}} f_{n, m^{\prime}}^{ \pm},
$$


together with the normalization condition

$$
\int \mathrm{d}^{2} x f_{n, m}^{ \pm}(x)=\sqrt{\frac{4 \pi}{B}} \delta_{n, m} .
$$

A better understanding of the physical meaning of these functions, and how the formulation of the field theory in terms of them is related to time-ordered perturbation theory, is currently lacking.

$\underline{\text { Unitarity and causality }}$

Each set of functions $f_{n, m}^{+}$and $f_{n, m}^{-}$on its own generates a complete basis for expansion of Schwartz fields $\phi$. The domain $\Phi$ is chosen such that both expansions can be taken simultaneously. Both matrix bases together imply stability and CT-invariance during matrix regularization. By stability we mean that expanding $\phi$ in the matrix bases $f_{n, m}^{ \pm}$and imposing the matrix regularization by cutting off these sums at some finite $N$ yields action functionals $S_{\Lambda}^{ \pm}[\phi]$, whose $\operatorname{sum} S_{\Lambda}[\phi]=\frac{1}{2}\left(S_{\Lambda}^{+}[\phi]+S_{\Lambda}^{-}[\phi]\right)$ is manifestly real. The CT-symmetry $\phi_{n, m}^{\mp}=C T \phi_{n, m}^{ \pm}$follows from the behaviours of the electric Landau wavefunctions under $T, P T$ and $C$ transformations given by

$$
\begin{aligned}
f_{m, n}^{ \pm}(-t, x) & =(-1)^{m-n} f_{n, m}^{ \pm}(t, x), \\
f_{m, n}^{ \pm}(-t,-x) & =(-1)^{m-n} f_{m, n}^{ \pm}(t, x), \\
f_{m, n}^{ \pm}(t, x)^{*} & =f_{n, m}^{\mp}(t, x)=(-1)^{m-n} f_{m, n}^{\mp}(-t, x) .
\end{aligned}
$$

Quantum duality

The proof of classical duality follows exactly the same route as in the euclidean case - it does not depend on the signature of the inner products used in Fourier transformation. At the quantum level, analogously to the euclidean case the regulated propagators in Minkowski space are obtained by replacing

$$
C^{ \pm}(n, m)=\left\langle\phi_{m, n}^{ \pm}{ }^{*} \phi_{m, n}^{\mp}\right\rangle
$$

with

$$
C_{\Lambda}^{ \pm}(n, m)=2 \mathrm{i}\left( \pm \mathrm{i} E_{n}+\mu^{2}\right)^{-1} F\left(\Lambda^{-2}\left|E_{n}+E_{m}\right|\right)
$$

Then the proof of quantum duality presented before carries through verbatim using these two sets of two-point functions. By multiplying (3.6) with $f_{m, n}^{ \pm}(x)^{*} f_{m, n}^{\mp}(y)$ and summing over all $n, m \in \mathbb{N}_{0}$ one obtains the position space representation of the propagator [15]. For the free Klein-Gordon field without electric field, after Fourier transformation this representation can be shown to possess the standard physical mass-shell poles [16].

Coupled complex two-matrix model representation

A non-trivial interacting two-matrix model now describes the minkowskian theory, whose action is generically rather involved [15]. With the same notation as before, it simplifies at the self-dual point to

$$
S[\phi]=\frac{1}{2} \sum_{ \pm} \operatorname{Tr}\left( \pm \phi_{ \pm}^{\dagger} \mathrm{i} \mathcal{B} \phi_{\mp}+\mu^{2} \phi_{ \pm}^{\dagger} \phi_{\mp}+\tilde{g}^{2}\left(\phi_{ \pm}^{\dagger} \phi_{\mp}\right)^{2}\right)
$$

This action possesses a much larger $G L(\infty) \times G L(\infty)$ symmetry $\phi_{ \pm} \longmapsto \phi_{ \pm} U_{ \pm}, \phi_{ \pm}^{\dagger} \longmapsto U_{\mp}^{-1} \phi_{ \pm}^{\dagger}$, and also the discrete CT-symmetry

$$
\left(\phi_{ \pm}, \phi_{ \pm}^{\dagger}\right) \longmapsto\left(\phi_{\mp}, \phi_{\mp}^{\dagger}\right), \theta \longmapsto-\theta .
$$


This two-matrix model representation clearly demonstrates that the Minkowski theory is not simply a Wick rotation of the euclidean theory.

\subsection{Derivations}

We will now sketch how these results are obtained, including a description of the configuration space $\Phi$. Just like the analysis of the standard Landau problem, and hence the duality covariant field theory in euclidean signature, is related to the harmonic oscillator, the model in Minkowski signature is related to the inverted harmonic oscillator whose hamiltonian is given by

$$
H=\frac{1}{2}\left(p^{2}-\omega^{2} q^{2}\right),
$$

where $\omega \in \mathbb{R}$ and $(p, q)$ are canonically conjugate variables. The functional analytic properties of the corresponding quantum hamiltonian are described in [17]. It is related to the usual harmonic oscillator hamiltonian by a complex scaling, which is a non-unitary similarity transformation which sends $\omega \longrightarrow \pm \mathrm{i} \omega$. The first quantized operator $\hat{H}$ corresponding to (3.10) is symmetric on a suitable domain in $L^{2}(\mathbb{R})$ with spectrum $\operatorname{Spec}(\hat{H})=\mathbb{R}$. In fact it admits a one-parameter family of self-adjoint extensions. This parameter should have some significance within the context of our duality covariant quantum field theory, but for simplicity we fix a self-adjoint extension and simply work with that. The relation between the classical hamiltonian $H$, the quantum hamiltonian $\hat{H}$, and the differential operator $D_{\mu}^{2}$ is established via the star product and the Wigner transform, defined for a rank one operator $\hat{\boldsymbol{\phi}}=|\psi\rangle\langle\varphi| \in L^{2}(\mathbb{R}) \otimes L^{2}(\mathbb{R})^{\vee}$ by

$$
\mathrm{W}(\hat{\phi})(t, x)=\frac{1}{2 \pi} \int_{\mathbb{R}} \mathrm{d} k \mathrm{e}^{\mathrm{i} k x}\langle t-\theta k / 2 \mid \psi\rangle\langle\varphi \mid t+\theta k / 2\rangle .
$$

For every function $f(x)=\mathbf{W}(\hat{f})(x)$ one has

$$
D_{\mu}^{2} f(x)=H \star f(x)=\mathrm{W}(\hat{H} \hat{f})(x) .
$$

Thus instead of working with $D_{\mu}^{2}$ operating on a set of fields, we will work with $\hat{H}$ acting on a suitable quantum mechanical Hilbert space.

The hamiltonian $\hat{H}$ also has a set of generalized eigenfunctions with imaginary eigenvalues. They occur as residues of the original eigenfunctions analytically continued to the complex energy plane. By closing the contour of integration in the eigenfunction expansion of a wavefunction appropriately, we pick up these states and obtain the analog of the discrete expansion in Landau wavefunctions. These techniques are analogous to those of the Bohm-Gadella theory of resonant states in quantum mechanics, wherein the instabilities mentioned above describe nuclear decay phenomena. The inverted harmonic oscillator potential and its resonance expansion also defines the analytic continuation of the Grosse-Wulkenhaar model to Minkowski signature. To explore the renormalization in this case, one needs to establish suitable decay properties of the free propagators in the matrix basis analogous to the euclidean case [5]. The properties of these Green's functions are currently under investigation [16].

Let us now explain the concepts introduced above. The mathematical setting we need is the extension of the notion of Hilbert space to that of a rigged Hilbert space (also known in the literature as a Gel'fand triple), which is a triple of spaces

$$
\Phi \subset \mathcal{H} \subset \Phi^{\prime}
$$

where $\Phi$ is a dense nuclear subspace of a Hilbert space $\mathcal{H}$ with dual $\Phi^{\prime}$, the space of continuous linear functionals $\Phi \rightarrow \mathbb{C}$. If $A \in \operatorname{End}(\mathcal{H})$ is self-adjoint on $\Phi$, then we can define its action on $\Phi^{\prime}$ 
using the dual pairing. A vector $F_{\lambda} \in \Phi^{\prime}$ is then said to be a generalized eigenvector of $A$ with generalized eigenvalue $\lambda \in \mathbb{C}$ if

$$
\left\langle A F_{\lambda} \mid \phi\right\rangle:=\left\langle F_{\lambda} \mid A \phi\right\rangle=\lambda\left\langle F_{\lambda} \mid \phi\right\rangle
$$

holds for all $\phi \in \Phi$. The Gel'fand-Maurin theorem asserts that for any $\phi \in \Phi$, there exists $F_{\lambda} \in \Phi^{\prime}$ such that there is an expansion

$$
\phi=\int_{\operatorname{Spec}(A)} \mathrm{d} \mu(\lambda) F_{\lambda}\left\langle F_{\lambda} \mid \phi\right\rangle
$$

where $\mathrm{d} \mu$ is discrete measure on the discrete part of the spectrum of $A$ and Lebesgue measure on the continuous part.

For the example of the inverted harmonic oscillator, the rigged Hilbert space is

$$
\mathcal{S}(\mathbb{R}) \subset L^{2}(\mathbb{R}) \subset \mathcal{S}^{\prime}(\mathbb{R}),
$$

where $\mathcal{S}(\mathbb{R})$ is the topological vector space of Schwartz functions on $\mathbb{R}$ (with the usual semi-norm topology) and $\mathcal{S}^{\prime}(\mathbb{R})$ is the space of tempered distributions on $\mathbb{R}$. By parity invariance, each eigenvalue $\mathcal{E} \in \operatorname{Spec}(\hat{H})=\mathbb{R}$ corresponds to two-fold degenerate eigenfunctions $\chi_{ \pm}^{\mathcal{E}}, \eta_{ \pm}^{\mathcal{E}} \in \mathcal{S}^{\prime}(\mathbb{R})$ which after rescaling $B \rightarrow B / 2$ are given explicitly by

$$
\begin{aligned}
\chi_{ \pm}^{\mathcal{E}}(q) & =\frac{C}{\sqrt{2 \pi B}} \mathrm{i}^{\frac{\nu}{2}+\frac{1}{4}} \Gamma(\nu+1) D_{-\nu-1}(\mp \sqrt{-2 \mathrm{i} B} q) \\
\eta_{ \pm}^{\mathcal{E}}(q) & =\frac{C}{\sqrt{2 \pi B}} \mathrm{i}^{\frac{\nu}{2}+\frac{1}{4}} \Gamma(-\nu) D_{\nu}(\mp \sqrt{2 \mathrm{i} B} q)
\end{aligned}
$$

where $C$ is a numerical constant, $\nu=-\mathrm{i} \frac{\mathcal{E}}{B}-\frac{1}{2}$, and $D_{\nu}(z)$ are parabolic cylinder functions. Only two of them are linearly independent, so for any $\phi \in \mathcal{S}(\mathbb{R})$ the Gel'fand-Maurin theorem gives a pair of expansions

$$
\phi(q)=\sum_{ \pm} \int_{\mathbb{R}} \mathrm{d} \mathcal{E} \chi_{ \pm}^{\mathcal{E}}(q)\left\langle\chi_{ \pm}^{\mathcal{E}} \mid \phi\right\rangle=\sum_{ \pm} \int_{\mathbb{R}} \mathrm{d} \mathcal{E} \eta_{ \pm}^{\mathcal{E}}(q)\left\langle\eta_{ \pm}^{\mathcal{E}} \mid \phi\right\rangle .
$$

The oscillator hamiltonian $\hat{H}$ also has generalized eigenfunctions $f_{n}^{ \pm}$with discrete eigenvalues $\pm \mathrm{i} B\left(n+\frac{1}{2}\right), n=0,1, \ldots$, occuring as residues of $\chi_{ \pm}^{\mathcal{E}} / \eta_{ \pm}^{\mathcal{E}}$ in the lower / upper complex half-plane. Then in a suitable domain $\phi \in \Phi \subset \mathcal{S}(\mathbb{R})$, an application of the residue theorem to the energy integrals in (3.18) gives the respective resonance expansions

$$
\phi(q)=\sum_{n=0}^{\infty} f_{n}^{-}(q)\left\langle f_{n}^{+} \mid \phi\right\rangle=\sum_{n=0}^{\infty} f_{n}^{+}(q)\left\langle f_{n}^{-} \mid \phi\right\rangle .
$$

The choice of configuration space $\Phi$ must ensure that both integrals over $\mathcal{E}$ in (3.18) can be extended to closed contour integrals for which the residue theorem applies in the usual way and such that the resonance expansions in (3.19) converge. In particular, it consists of fields $\phi$ such that $\chi_{ \pm}^{\mathcal{E}}(q)\left\langle\chi_{ \pm}^{\mathcal{E}} \mid \phi\right\rangle$ vanishes uniformly almost everywhere as $\mathcal{E}$ tends to infinity in the lower complex halfplane, and $\eta_{ \pm}^{\mathcal{E}}(q)\left\langle\eta_{ \pm}^{\mathcal{E}} \mid \phi\right\rangle$ vanishes uniformly almost everywhere as $\mathcal{E}$ tends to infinity in the upper complex half-plane, together with the analogous vanishing requirements on the scalar products $\left\langle\psi \mid \chi_{ \pm}^{\mathcal{E}}\right\rangle\left\langle\chi_{ \pm}^{\mathcal{E}} \mid \phi\right\rangle$ and $\left\langle\psi \mid \eta_{ \pm}^{\mathcal{E}}\right\rangle\left\langle\eta_{ \pm}^{\mathcal{E}} \mid \phi\right\rangle$ for all $\phi, \psi \in \Phi$. For this, consider the rigged Hilbert space

$$
\mathcal{S}_{\alpha}^{\alpha}(\mathbb{R}) \subset L^{2}(\mathbb{R}) \subset \mathcal{S}_{\alpha}^{\alpha}(\mathbb{R})^{\prime},
$$


where $\mathcal{S}_{\alpha}^{\alpha}(\mathbb{R})$ is a Gel'fand-Shilov space with $\alpha \geq \frac{1}{2}$, and its dual $\mathcal{S}_{\alpha}^{\alpha}(\mathbb{R})^{\prime}$ is a space of tempered ultra-distributions of Roumieu type. These Gel'fand-Shilov spaces contain entire functions $\phi(q)$ on $\mathbb{C}$ restricted to $\mathbb{R}$, with $L^{\infty}$-norms obeying $\left\|q^{m} \partial_{q}^{n} \phi\right\|_{\infty} \leq C M^{n+m} n^{\alpha n} m^{\alpha m}$ for all $n, m \in \mathbb{N}_{0}$ with some constant $C$ and given $M$. They form dense subspaces of Schwartz space $\mathcal{S}_{\alpha}^{\alpha}(\mathbb{R}) \subset$ $\mathcal{S}(\mathbb{R})=\mathcal{S}_{\infty}^{\infty}(\mathbb{R})$ which are closed under Fourier transformation and the star-product [18, 19], and which are generated by the basis of harmonic oscillator wavefunctions [20]. They are thus natural configuration spaces for duality covariant noncommutative field theories. The boundedness properties of these functions, together with the asymptotic behaviours of the parabolic cylinder functions and the gamma-functions, appear to be sufficient to ensure that the integrands in (3.18) and all pertinent pairing factors vanish appropriately $[15,16]$.

The mapping from functions on $\mathbb{R}$ in (3.19) to fields on $\mathbb{R}^{2}$ in (3.1) is given by applying the Wigner transformation $\mathrm{W}: \mathcal{S}_{\alpha}^{\alpha}(\mathbb{R}) \otimes \mathcal{S}_{\alpha}^{\alpha}(\mathbb{R})^{\vee} \longrightarrow \mathcal{S}_{\alpha}^{\alpha}\left(\mathbb{R}^{2}\right)$ to a rank one operator $\hat{\phi}=|\psi\rangle\langle\varphi|$ using the integral formula (3.11). Expanding $\hat{\phi}$ in parabolic cylinder functions,

$$
\begin{aligned}
\hat{\phi} & =\sum_{s, s^{\prime}= \pm} \int_{\mathbb{R}} \mathrm{d} \mathcal{E} \int_{\mathbb{R}} \mathrm{d} \mathcal{E}^{\prime}\left|\chi_{s}^{\mathcal{E}}\right\rangle\left\langle\chi_{s}^{\mathcal{E}} \mid \psi\right\rangle\left\langle\varphi \mid \eta_{s^{\prime}}^{\mathcal{E}^{\prime}}\right\rangle\left\langle\eta_{s^{\prime}}^{\mathcal{E}^{\prime}}\right| \\
& =\sum_{s, s^{\prime}= \pm} \int_{\mathbb{R}} \mathrm{d} \mathcal{E} \int_{\mathbb{R}} \mathrm{d} \mathcal{E}^{\prime}\left|\eta_{s}^{\mathcal{E}}\right\rangle\left\langle\eta_{s}^{\mathcal{E}} \mid \psi\right\rangle\left\langle\varphi \mid \chi_{s^{\prime}}^{\mathcal{E}^{\prime}}\right\rangle\left\langle\chi_{s^{\prime}}^{\mathcal{E}^{\prime}}\right|
\end{aligned}
$$

one can read off the respective resonance expansions (3.1) from (3.18) and (3.19). Using this mapping one can also explicitly compute the electric Landau wavefunctions [15]

$$
\begin{aligned}
f_{m, n}^{ \pm}(t, x)= & (-1)^{\min (m, n)} \sqrt{\frac{B}{\pi}} \sqrt{\frac{\min (m, n) !}{\max (m, n) !}}( \pm \mathrm{i} B)^{|m-n| / 2} \\
& \times \mathrm{e}^{\mp \mathrm{i} B x_{+} x_{-} / 2} x_{\mp \operatorname{mgn}(m-n)}^{|m-n|} L_{\min (m, n)}^{|m-n|}\left( \pm \mathrm{i} B x_{+} x_{-}\right),
\end{aligned}
$$

where $x_{ \pm}=t \pm x$ and

$$
L_{n}^{\alpha}(z)=\sum_{q=0}^{n}\left(\begin{array}{l}
\alpha+n \\
n-q
\end{array}\right) \frac{(-z)^{q}}{q !}
$$

are the generalized Laguerre polynomials. Using these explicit forms one can straightforwardly derive all properties of $f_{m, n}^{ \pm}$stated above.

Essential for the proof of orthogonality is the occurance of the phase factors $\mathrm{e}^{\mp \mathrm{i} B x_{+} x_{-} / 2}$ in (3.22), which generate derivatives via the identity $\int_{\mathbb{R}} \mathrm{d} x_{-}\left(x_{-}\right)^{p} \mathrm{e}^{\mp \mathrm{i} x_{+} x_{-}}=2 \pi\left( \pm \mathrm{i} \partial_{+}\right)^{p} \delta\left(x_{+}\right)$ and ensure that the integrals over $\left(x_{+}, x_{-}\right) \in \mathbb{R}^{2}$ converge. This is the reason why only the $L^{2}$-inner products $\left(f_{m, n}^{\mp}, f_{k, l}^{ \pm}\right)_{L^{2}}$ are permitted, since this exponential factor is absent for the other combinations. For the same reason only terms with equal powers of $x_{+}$and $x_{-}$survive the integration. After some algebra one then readily verifies the orthogonality relations

$$
\left(f_{m, n}^{\mp}, f_{k, l}^{ \pm}\right)_{L^{2}}=\int \mathrm{d} x \mathrm{~d} t f_{n, m}^{ \pm}(t, x) f_{k, l}^{ \pm}(t, x)=\delta_{n, l} \delta_{m, k} .
$$

The normalization relation (3.4) is computed in an analogous way.

\section{Note added}

After this paper was submitted for publication, the preprint [21] appeared with some critiques of our approach in [15]. In particular, a counterexample to our Theorem 4.2 is found, casting doubt on our choice of domain $\Phi$. While this critique is fully justified, we have amended our calculations, 
and found that both our usage of and conclusions infered from the matrix basis are still in fact valid. Briefly, one splits the Minkowski space action functional $S_{\mathrm{M}}$ at $g=0$ into two parts as

$$
S_{\mathrm{M}}=\frac{1}{2}\left(S^{(+\epsilon)}+S^{(-\epsilon)}\right), \quad S^{( \pm \epsilon)}=S_{\mathrm{M}} \pm \mathrm{i} \tan (\epsilon) S_{\mathrm{E}}
$$

where $0<\epsilon<\frac{\pi}{2}$ and $S_{\mathrm{E}}$ is the euclidean action at $g=0$. In the same manner that $S_{\mathrm{E}}$ and $S_{\mathrm{M}}$ can be related to the harmonic and inverted harmonic oscillators, respectively, the actions $S^{( \pm \epsilon)}$ are related to the complex harmonic oscillator with hamiltonian $\frac{1}{2}\left(p^{2}-\mathrm{e}^{\mp 2 \mathrm{i} \epsilon} q^{2}\right)$. In contrast to the inverted harmonic oscillator, this family of hamiltonians have discrete eigenvalues given by the harmonic oscillator spectrum scaled by $\pm \mathrm{i} \mathrm{e}^{\mp \mathrm{i} \epsilon}$, while its eigenfunctions span $L^{2}(\mathbb{R})$ and have the usual star-product projection property. Path integral quantisation can now be easily carried out, leading to the results summarised above in the limit $\epsilon \rightarrow 0$. The details of our modified analysis, together with various applications to the computation of propagators in our model, will appear in a forthcoming paper [16]. As stressed in $[4,15]$ and reviewed above, the existence of the matrix basis is the crux of the proof of duality covariance at the quantum level. It also led to the original proof [5] of renormalizability of the Grosse-Wulkenhaar model. From our point of view, the matrix basis is taken as part of the definition of the (regularized) duality covariant quantum field theory. We do not know how to establish quantum duality using the basis of continuum eigenfunctions, on which the analysis of [21] is (partly) based.

\section{Acknowledgments}

We thank A.P. Balachandran, E.B. Davies, K. Fredenhagen, H. Grosse, H. Steinacker and J. Zahn for helpful comments, discussions and correspondence. The work of RJS is supported in part by grant ST/G000514/1 "String Theory Scotland" from the UK Science and Technology Facilities Council.

\section{References}

[1] R.J. Szabo, Phys. Rept. 378 (2003) 207 [arXiv:hep-th/0109162].

[2] S. Minwalla, M. van Raamsdonk and N. Seiberg, JHEP 0002 (2000) 020 [arXiv:hepth/9912072].

[3] H. Grosse and M. Wohlgenannt, Nucl. Phys. B 748 (2006) 473 [arXiv:hep-th/0507030].

[4] E. Langmann and R.J. Szabo, Phys. Lett. B 533 (2002) 168 [arXiv:hep-th/0202039].

[5] H. Grosse and R. Wulkenhaar, Commun. Math. Phys. 256 (2005) 305 [arXiv:hep-th/0401128].

[6] E. Langmann, R.J. Szabo and K. Zarembo, JHEP 0401 (2004) 017 [arXiv:hep-th/0308043].

[7] H. Grosse and R. Wulkenhaar, Eur. Phys. J. C 35 (2004) 277 [arXiv:hep-th/0402093].

[8] V. Rivasseau, Sém. Poincaré X (2007) 15 [arXiv:hep-th/0702068].

[9] R. Gurau and O.J. Rosten, JHEP 0907 (2009) 064 [arXiv:0902.4888 [hep-th]].

[10] P. Bieliavsky, R. Gurau and V. Rivasseau, J. Noncommut. Geom. 3 (2009) 99 [arXiv:0806.4255 [hep-th]].

[11] D. Bahns, S. Doplicher, K. Fredenhagen and G. Piacitelli, Phys. Lett. B 533 (2002) 178 [arXiv:hep-th/0201222]. 
[12] Y. Liao and K. Sibold, Eur. Phys. J. C 25 (2002) 469 [arXiv:hep-th/0205269].

[13] C.-h. Rim and J.H. Yee, Phys. Lett. B 574 (2003) 111 [arXiv:hep-th/0205193].

[14] D. Bahns, arXiv:0908.4537 [math-ph].

[15] A. Fischer and R.J. Szabo, JHEP 0902 (2009) 031 [arXiv:0810.1195 [hep-th]].

[16] A. Fischer and R.J. Szabo, to appear.

[17] D. Chruscinski, J. Math. Phys. 45 (2004) 841 [arXiv:math-ph/0307047].

[18] M.A. Soloviev, Theor. Math. Phys. 153 (2007) 1351 [arXiv:0708.0811 [math-ph]].

[19] M. Chaichian, M. Mnatsakanova, A. Tureanu and Yu. Vernov, JHEP 0809 (2008) 125 [arXiv:0706.1712 [hep-th]].

[20] Z. Lozanov-Crvenković and D. Perišić, Novi Sad J. Math. 37 (2007) 129 [arXiv:quant$\mathrm{ph} / 0701041]$.

[21] J. Zahn, arXiv:1005.0541 [hep-th]. 